Tables S1. Values of $\mathrm{pH}$ (total scale), temperature $\left({ }^{\circ} \mathrm{C}\right)$, salinity $\left(\mathrm{g} \mathrm{kg}^{-1}\right)$, dissolved oxygen $\left(\mathrm{DO} ; \mathrm{mg} \mathrm{L}^{-}\right.$ $\left.{ }^{1}\right), \mathrm{pCO}_{2}(\mu \mathrm{atm})$, total alkalinity $\left(\mu \mathrm{mol} \mathrm{kgSW}{ }^{-1}\right)$, total DIC $\left(\mu \mathrm{mol} \mathrm{kgSW}{ }^{-1}\right), \mathrm{HCO}_{3}^{-}\left(\mu \mathrm{mol} \mathrm{kgSW}{ }^{-1}\right)$, $\mathrm{CO}_{3}{ }^{2-}\left(\mu \mathrm{mol} \mathrm{kgSW}{ }^{-1}\right), \mathrm{OH}^{-}\left(\mu \mathrm{mol} \mathrm{kgSW}{ }^{-1}\right), \Omega_{\text {calcite, }}$ and $\Omega_{\text {aragonite }}$ for June through November experiments. Values represent means \pm standard error.

Mercenaria mercenaria - smaller cohorts

\begin{tabular}{rcccc} 
Parameter & Control & Ulva & $\mathrm{CO}_{2}$ & $\mathrm{CO}_{2} /$ Ulva \\
\hline $\mathrm{pH}$ & $8.11 \pm 0.04$ & $8.13 \pm 0.04$ & $7.47 \pm 0.03$ & $7.49 \pm 0.03$ \\
Temperature & $20.9 \pm 0.5$ & $20.9 \pm 0.5$ & $20.9 \pm 0.4$ & $20.9 \pm 0.4$ \\
$\mathrm{DO}$ & $9.41 \pm 0.13$ & $9.29 \pm 0.15$ & $9.26 \pm 0.09$ & $9.13 \pm 0.11$ \\
Salinity & $30.0 \pm 0.6$ & $30.0 \pm 0.6$ & $30.0 \pm 0.6$ & $30.0 \pm 0.6$ \\
$\mathrm{pCO}_{2}$ & $309 \pm 27$ & $290 \pm 33$ & $1580 \pm 112$ & $1513 \pm 99$ \\
Total alkalinity & $2068 \pm 23$ & $2066 \pm 19$ & $2023 \pm 9$ & $2019 \pm 7$ \\
Total DIC & $1815 \pm 200$ & $1790 \pm 197$ & $2004 \pm 220$ & $1992 \pm 219$ \\
$\mathrm{HCO}_{3}{ }^{2-}$ & $1628 \pm 15$ & $1598 \pm 18$ & $1905 \pm 10$ & $1894 \pm 10$ \\
$\mathrm{CO}_{3}{ }^{-}$ & $180 \pm 14$ & $183 \pm 19$ & $48 \pm 4$ & $49 \pm 4$ \\
$\mathrm{OH}^{-}$ & $4.40 \pm 0.39$ & $4.75 \pm 0.32$ & $1.17 \pm 0.09$ & $1.25 \pm 0.07$ \\
$\Omega_{\text {calcite }}$ & $4.40 \pm 0.39$ & $4.75 \pm 0.32$ & $0.76 \pm 0.06$ & $1.25 \pm 0.07$ \\
$\Omega_{\text {aragonite }}$ & $2.83 \pm 0.25$ & $3.06 \pm 0.21$ & & $0.80 \pm 0.05$
\end{tabular}

Mercenaria mercenaria - larger cohorts

\begin{tabular}{rcccc} 
Parameter & Control & Ulva & $\mathrm{CO}_{2}$ & $\mathrm{CO}_{2} /$ Ulva \\
\hline $\mathrm{pH}$ & $7.91 \pm 0.07$ & $7.95 \pm 0.08$ & $7.34 \pm 0.02$ & $7.36 \pm 0.02$ \\
Temperature & $21.1 \pm 0.5$ & $21.1 \pm 0.5$ & $21.1 \pm 0.5$ & $21.1 \pm 0.5$ \\
$\mathrm{DO}$ & $9.06 \pm 0.15$ & $9.07 \pm 0.14$ & $9.21 \pm 0.17$ & $8.95 \pm 0.09$ \\
Salinity & $30.0 \pm 0.6$ & $30.0 \pm 0.6$ & $30.0 \pm 0.6$ & $30.0 \pm 0.6$ \\
$\mathrm{pCO}_{2}$ & $435 \pm 86$ & $338 \pm 74$ & $1541 \pm 73$ & $1634 \pm 68$ \\
Total alkalinity & $1663 \pm 24$ & $1450 \pm 8$ & $1436 \pm 3$ & $1601 \pm 4$ \\
Total DIC & $1527 \pm 168$ & $1293 \pm 142$ & $1444 \pm 159$ & $1606 \pm 177$ \\
$\mathrm{HCO}_{3}{ }^{2-}$ & $1415 \pm 12$ & $1192 \pm 12$ & $1369 \pm 1$ & $1524 \pm 1$ \\
$\mathrm{CO}_{3}{ }^{-}$ & $98 \pm 15$ & $90 \pm 14$ & $0.87 \pm 0.05$ & $29 \pm 1$ \\
$\mathrm{OH}^{-}$ & $3.09 \pm 0.46$ & $3.86 \pm 0.23$ & $0.62 \pm 0.02$ & $0.90 \pm 0.07$ \\
$\Omega_{\text {calcite }}$ & $2.34 \pm 0.34$ & $2.43 \pm 0.12$ & $0.40 \pm 0.01$ & $0.73 \pm 0.03$ \\
$\Omega_{\text {aragonite }}$ & $1.50 \pm 0.22$ & $1.57 \pm 0.08$ & & $0.47 \pm 0.02$
\end{tabular}


Crassostrea virginica - smaller cohorts

\begin{tabular}{rcccc} 
Parameter & Control & Ulva & $\mathrm{CO}_{2}$ & $\mathrm{CO}_{2} /$ Ulva \\
\hline $\mathrm{pH}$ & $8.09 \pm 0.05$ & $8.21 \pm 0.11$ & $7.44 \pm 0.03$ & $7.46 \pm 0.05$ \\
Temperature & $21.6 \pm 0.6$ & $21.6 \pm 0.6$ & $21.6 \pm 0.7$ & $21.6 \pm 0.6$ \\
$\mathrm{DO}$ & $9.23 \pm 0.11$ & $8.97 \pm 0.08$ & $9.13 \pm 0.09$ & $9.02 \pm 0.10$ \\
Salinity & $30.0 \pm 0.6$ & $30.0 \pm 0.6$ & $30.0 \pm 0.6$ & $30.0 \pm 0.6$ \\
$\mathrm{pCO}_{2}$ & $287 \pm 37$ & $210 \pm 55$ & $1649 \pm 120$ & $1513 \pm 158$ \\
Total alkalinity & $1830 \pm 22$ & $1832 \pm 68$ & $1971 \pm 8$ & $1895 \pm 13$ \\
Total DIC & $1600 \pm 176$ & $1541 \pm 170$ & $1957 \pm 215$ & $1875 \pm 206$ \\
$\mathrm{HCO}_{3}{ }^{2-}$ & $1437 \pm 13$ & $1343 \pm 44$ & $1860 \pm 1$ & $452 \pm 1$ \\
$\mathrm{CO}_{3}{ }^{-}$ & $154 \pm 14$ & $192 \pm 45$ & $1.15 \pm 0.09$ & $1.21 \pm 0.18$ \\
$\mathrm{OH}^{-}$ & $5.16 \pm 0.51$ & $6.93 \pm 1.99$ & $1.11 \pm 0.07$ & $1.12 \pm 0.13$ \\
$\Omega_{\text {calcite }}$ & $3.84 \pm 0.35$ & $4.78 \pm 1.13$ & $0.72 \pm 0.05$ & $0.72 \pm 0.08$
\end{tabular}

Crassostrea virginica - larger cohorts

\begin{tabular}{rcccc} 
Parameter & Control & Ulva & $\mathrm{CO}_{2}$ & $\mathrm{CO}_{2} /$ Ulva \\
\hline $\mathrm{pH}$ & $7.87 \pm 0.05$ & $7.92 \pm 0.06$ & $7.30 \pm 0.03$ & $7.31 \pm 0.03$ \\
Temperature & $20.8 \pm 0.6$ & $20.8 \pm 0.5$ & $20.9 \pm 0.5$ & $20.9 \pm 0.5$ \\
$\mathrm{DO}$ & $8.97 \pm 0.17$ & $8.56 \pm 0.74$ & $9.02 \pm 0.16$ & $8.97 \pm 0.16$ \\
Salinity & $29.0 \pm 0.6$ & $29.1 \pm 0.6$ & $28.9 \pm 0.6$ & $29.0 \pm 0.6$ \\
$\mathrm{pCO}_{2}$ & $398 \pm 49$ & $363 \pm 55$ & $1945 \pm 150$ & $1825 \pm 144$ \\
Total alkalinity & $1374 \pm 15$ & $1459 \pm 20$ & $1647 \pm 3$ & $1576 \pm 7$ \\
Total DIC & $1265 \pm 139$ & $1327 \pm 146$ & $1673 \pm 184$ & $1592 \pm 175$ \\
$\mathrm{HCO}_{3}{ }^{-}$ & $1180 \pm 7$ & $1230 \pm 10$ & $1584 \pm 2$ & $25 \pm 1$ \\
$\mathrm{CO}_{3}{ }^{-}$ & $71 \pm 8$ & $85 \pm 11$ & $0.74 \pm 0.07$ & $26 \pm 2$ \\
$\mathrm{OH}^{-}$ & $2.79 \pm 0.35$ & $3.20 \pm 0.47$ & $0.63 \pm 0.02$ & $0.79 \pm 0.06$ \\
$\Omega_{\text {calcite }}$ & $1.78 \pm 0.21$ & $2.13 \pm 0.29$ & $0.41 \pm 0.02$ & $0.64 \pm 0.05$ \\
$\Omega_{\text {aragonite }}$ & $1.14 \pm 0.13$ & $1.37 \pm 0.18$ & $0.42 \pm 0.03$
\end{tabular}

Argopecten irradians - smaller cohorts

\begin{tabular}{rcccc} 
Parameter & Control & Ulva & $\mathrm{CO}_{2}$ & $\mathrm{CO}_{2} /$ Uva \\
\hline $\mathrm{pH}$ & $7.94 \pm 0.03$ & $8.01 \pm 0.07$ & $7.35 \pm 0.03$ & $7.37 \pm 0.04$ \\
Temperature & $21.8 \pm 0.9$ & $21.8 \pm 0.9$ & $21.8 \pm 0.9$ & $21.7 \pm 0.9$ \\
$\mathrm{DO}$ & $9.16 \pm 0.16$ & $9.00 \pm 0.16$ & $9.00 \pm 0.16$ & $9.01 \pm 0.16$ \\
Salinity & $30.0 \pm 0.6$ & $30.0 \pm 0.6$ & $30.0 \pm 0.6$ & $30.0 \pm 0.6$ \\
$\mathrm{pCO}_{2}$ & $422 \pm 28$ & $368 \pm 62$ & $1898 \pm 137$ & $1938 \pm 196$ \\
Total alkalinity & $1778 \pm 12$ & $1839 \pm 36$ & $1816 \pm 7$ & $1947 \pm 11$ \\
Total DIC & $1615 \pm 178$ & $1644 \pm 181$ & $1824 \pm 201$ & $1951 \pm 215$ \\
$\mathrm{HCO}_{3}^{-}$ & $1489 \pm 7$ & $1499 \pm 21$ & $1730 \pm 2$ & $1852 \pm 2$ \\
$\mathrm{CO}_{3}{ }^{-}$ & $112 \pm 8$ & $133 \pm 23$ & $0.93 \pm 0.07$ & $38 \pm 4$ \\
$\mathrm{OH}^{-}$ & $3.58 \pm 0.31$ & $4.33 \pm 0.92$ & $0.84 \pm 0.05$ & $0.98 \pm 0.09$ \\
$\Omega_{\text {calcite }}$ & $2.80 \pm 0.19$ & $3.32 \pm 0.57$ & $0.54 \pm 0.03$ & $0.94 \pm 0.09$ \\
$\Omega_{\text {aragonite }}$ & $1.80 \pm 0.12$ & $2.14 \pm 0.37$ & & $0.61 \pm 0.06$
\end{tabular}


Argopecten irradians - larger cohorts

\begin{tabular}{rcccc} 
Parameter & Control & Ulva & $\mathrm{CO}_{2}$ & $\mathrm{CO}_{2} /$ Ulva \\
\hline $\mathrm{pH}$ & $7.96 \pm 0.02$ & $7.99 \pm 0.03$ & $7.34 \pm 0.04$ & $7.36 \pm 0.04$ \\
Temperature & $21.2 \pm 0.6$ & $21.1 \pm 0.5$ & $21.3 \pm 0.5$ & $21.3 \pm 0.6$ \\
$\mathrm{DO}$ & $8.86 \pm 0.18$ & $9.06 \pm 0.32$ & $8.96 \pm 0.21$ & $9.20 \pm 0.17$ \\
Salinity & $30.4 \pm 0.6$ & $30.4 \pm 0.6$ & $30.5 \pm 0.6$ & $30.4 \pm 0.6$ \\
$\mathrm{pCO}_{2}$ & $405 \pm 18$ & $396 \pm 33$ & $2018 \pm 171$ & $1962 \pm 159$ \\
Total alkalinity & $1803 \pm 7$ & $1888 \pm 14$ & $1899 \pm 10$ & $1919 \pm 9$ \\
Total DIC & $1633 \pm 180$ & $1702 \pm 187$ & $1911 \pm 210$ & $1926 \pm 212$ \\
$\mathrm{HCO}_{3}{ }^{-}$ & $1503 \pm 4$ & $1560 \pm 7$ & $1812 \pm 2$ & $35 \pm 3$ \\
$\mathrm{CO}_{3}{ }^{-}$ & $117 \pm 5$ & $129 \pm 8$ & $0.91 \pm 0.08$ & $36 \pm 3$ \\
$\mathrm{OH}^{-}$ & $3.65 \pm 0.21$ & $3.85 \pm 0.28$ & $0.85 \pm 0.07$ & $0.95 \pm 0.08$ \\
$\Omega_{\text {calcite }}$ & $2.90 \pm 0.11$ & $3.19 \pm 0.21$ & $0.55 \pm 0.05$ & $0.89 \pm 0.07$ \\
$\Omega_{\text {aragonite }}$ & $1.87 \pm 0.07$ & $2.06 \pm 0.13$ & & $0.58 \pm 0.04$
\end{tabular}

\section{Mytilus edulis}

\begin{tabular}{rcccc} 
Parameter & Control & Ulva & $\mathrm{CO}_{2}$ & $\mathrm{CO}_{2} /$ Ulva \\
\hline $\mathrm{pH}$ & $7.98 \pm 0.11$ & $8.00 \pm 0.10$ & $7.36 \pm 0.07$ & $7.37 \pm 0.07$ \\
Temperature & $21.7 \pm 0.9$ & $21.7 \pm 0.9$ & $21.7 \pm 1.0$ & $21.7 \pm 1.0$ \\
$\mathrm{DO}$ & $9.12 \pm 0.17$ & $9.08 \pm 0.14$ & $9.05 \pm 0.16$ & $9.09 \pm 0.13$ \\
Salinity & $31.0 \pm 0.6$ & $31.0 \pm 0.6$ & $31.0 \pm 0.6$ & $31.0 \pm 0.6$ \\
$\mathrm{pCO}_{2}$ & $357 \pm 85$ & $361 \pm 78$ & $1648 \pm 226$ & $1636 \pm 223$ \\
Total alkalinity & $1629 \pm 32$ & $1765 \pm 51$ & $1617 \pm 10$ & $1643 \pm 18$ \\
Total DIC & $1472 \pm 162$ & $1572 \pm 173$ & $1624 \pm 179$ & $1642 \pm 181$ \\
$\mathrm{HCO}_{3}{ }^{2-}$ & $1345 \pm 29$ & $1432 \pm 30$ & $1540 \pm 2$ & $1557 \pm 2$ \\
$\mathrm{CO}_{3}{ }^{-}$ & $103 \pm 19$ & $129 \pm 32$ & $0.90 \pm 0.13$ & $33 \pm 6$ \\
$\mathrm{OH}^{-}$ & $3.34 \pm 0.33$ & $4.40 \pm 1.44$ & $0.72 \pm 0.08$ & $1.02 \pm 0.26$ \\
$\Omega_{\text {calcite }}$ & $2.55 \pm 0.47$ & $3.19 \pm 0.81$ & $0.47 \pm 0.05$ & $0.81 \pm 0.16$ \\
$\Omega_{\text {aragonite }}$ & $1.65 \pm 0.31$ & $2.06 \pm 0.53$ & & $0.53 \pm 0.11$
\end{tabular}


Table S2. Two-way analyses of variance for $\Omega_{\text {calcite }}$ for smaller and larger cohorts of hard clams (Mercenaria mercenaria), Eastern oysters (Crassostrea virginica), bay scallops (Argopecten irradians), and blue mussels (Mytilus edulis). Asterisks next to p-values represent significant results.

\begin{tabular}{|c|c|c|c|c|c|c|}
\hline $\begin{array}{c}\text { Species (size } \\
\text { class) }\end{array}$ & $\begin{array}{c}\text { Source of } \\
\text { variation }\end{array}$ & DF & SS & MS & $\mathrm{F}$ & $P$ \\
\hline \multirow{5}{*}{$\begin{array}{l}\text { Mercenaria } \\
\text { mercenaria } \\
\quad \text { (small) }\end{array}$} & $\mathrm{CO}_{2}$ & 1 & 112.366 & 112.366 & 1559.605 & $<0.001 *$ \\
\hline & Ulva & 1 & 0.486 & 0.486 & 6.752 & $0.013^{*}$ \\
\hline & $\mathrm{CO}_{2} \times U l v a$ & 1 & 0.157 & 0.157 & 2.176 & 0.149 \\
\hline & Residual & 36 & 2.594 & 0.072 & & \\
\hline & Total & 39 & 115.403 & 2.959 & & \\
\hline \multirow{5}{*}{$\begin{array}{l}\text { Mercenaria } \\
\text { mercenaria } \\
\quad \text { (large) }\end{array}$} & $\mathrm{CO}_{2}$ & 1 & 22.839 & 22.839 & 849.289 & $<0.001 *$ \\
\hline & Ulva & 1 & 0.127 & 0.127 & 4.72 & $0.038^{*}$ \\
\hline & $\mathrm{CO}_{2} \times$ Ulva & 1 & 0.00227 & 0.00227 & 0.0844 & 0.773 \\
\hline & Residual & 29 & 0.78 & 0.0269 & & \\
\hline & Total & 32 & 23.695 & 0.74 & & \\
\hline \multirow{5}{*}{$\begin{array}{l}\text { Crassostrea } \\
\text { virginica } \\
\text { (small) }\end{array}$} & $\mathrm{CO}_{2}$ & 1 & 91.745 & 91.745 & 257.28 & $<0.001 *$ \\
\hline & Ulva & 1 & 2.022 & 2.022 & 5.669 & $0.023^{*}$ \\
\hline & $\mathrm{CO}_{2} \times$ Ulva & 1 & 1.956 & 1.956 & 5.485 & $0.026^{*}$ \\
\hline & Residual & 32 & 11.411 & 0.357 & & \\
\hline & Total & 35 & 107.134 & 3.061 & & \\
\hline \multirow{5}{*}{$\begin{array}{c}\text { Crassostrea } \\
\text { virginica } \\
\text { (large) }\end{array}$} & $\mathrm{CO}_{2}$ & 1 & 17.92 & 17.92 & 483.796 & $<0.001 *$ \\
\hline & Ulva & 1 & 0.33 & 0.33 & 8.902 & $0.005^{*}$ \\
\hline & $\mathrm{CO}_{2} \times U l v a$ & 1 & 0.287 & 0.287 & 7.741 & $0.008 *$ \\
\hline & Residual & 38 & 1.408 & 0.037 & & \\
\hline & Total & 41 & 20.161 & 0.492 & & \\
\hline \multirow{5}{*}{$\begin{array}{l}\text { Argopecten } \\
\text { irradians } \\
\text { (small) }\end{array}$} & $\mathrm{CO}_{2}$ & 1 & 37.555 & 37.555 & 406.711 & $<0.001^{*}$ \\
\hline & Ulva & 1 & 0.786 & 0.786 & 8.513 & $0.007 *$ \\
\hline & $\mathrm{CO}_{2} \times U l v a$ & 1 & 0.344 & 0.344 & 3.72 & 0.064 \\
\hline & Residual & 28 & 2.585 & 0.0923 & & \\
\hline & Total & 31 & 41.27 & 1.331 & & \\
\hline \multirow{5}{*}{$\begin{array}{l}\text { Argopecten } \\
\text { irradians } \\
\quad \text { (large) }\end{array}$} & $\mathrm{CO}_{2}$ & 1 & 61.356 & 61.356 & 3715.401 & $<0.001 *$ \\
\hline & Ulva & 1 & 0.366 & 0.366 & 22.184 & $<0.001 *$ \\
\hline & $\mathrm{CO}_{2} \times$ Ulva & 1 & 0.218 & 0.218 & 13.183 & $<0.001 *$ \\
\hline & Residual & 48 & 0.793 & 0.0165 & & \\
\hline & Total & 51 & 62.733 & 1.23 & & \\
\hline \multirow{5}{*}{$\begin{array}{l}\text { Mytilus } \\
\text { edulis }\end{array}$} & $\mathrm{CO}_{2}$ & 1 & 39.294 & 39.294 & 163.406 & $<0.001 *$ \\
\hline & Ulva & 1 & 1.16 & 1.16 & 4.822 & $0.035^{*}$ \\
\hline & $\mathrm{CO}_{2} \times U l v a$ & 1 & 0.661 & 0.661 & 2.749 & 0.107 \\
\hline & Residual & 32 & 7.695 & 0.24 & & \\
\hline & Total & 35 & 50.455 & 1.442 & & \\
\hline
\end{tabular}


Table S3. Two-way analyses of variance for $\Omega_{\text {aragonite }}$ for smaller and larger cohorts of hard clams (Mercenaria mercenaria), Eastern oysters (Crassostrea virginica), bay scallops (Argopecten irradians), and blue mussels (Mytilus edulis). Asterisks next to p-values represent significant results.

\begin{tabular}{|c|c|c|c|c|c|c|}
\hline $\begin{array}{c}\text { Species (size } \\
\text { class) }\end{array}$ & $\begin{array}{c}\text { Source of } \\
\text { variation }\end{array}$ & DF & SS & MS & $\mathrm{F}$ & $P$ \\
\hline \multirow{5}{*}{$\begin{array}{l}\text { Mercenaria } \\
\text { mercenaria } \\
\quad \text { (small) }\end{array}$} & $\mathrm{CO}_{2}$ & 1 & 48.218 & 48.218 & 1641.78 & $<0.001 *$ \\
\hline & Ulva & 1 & 0.187 & 0.187 & 6.359 & $0.016^{*}$ \\
\hline & $\mathrm{CO}_{2} \times U l v a$ & 1 & 0.0839 & 0.0839 & 2.856 & 0.099 \\
\hline & Residual & 38 & 1.116 & 0.0294 & & \\
\hline & Total & 41 & 50.024 & 1.22 & & \\
\hline \multirow{5}{*}{$\begin{array}{l}\text { Mercenaria } \\
\text { mercenaria } \\
\quad \text { (large) }\end{array}$} & $\mathrm{CO}_{2}$ & 1 & 9.474 & 9.474 & 848.148 & $<0.001 *$ \\
\hline & Ulva & 1 & 0.0539 & 0.0539 & 4.827 & $0.036^{*}$ \\
\hline & $\mathrm{CO}_{2} \times$ Ulva & 1 & 0.00078 & 0.00078 & 0.0697 & 0.794 \\
\hline & Residual & 29 & 0.324 & 0.0112 & & \\
\hline & Total & 32 & 9.83 & 0.307 & & \\
\hline \multirow{5}{*}{$\begin{array}{l}\text { Crassostrea } \\
\text { virginica } \\
\text { (small) }\end{array}$} & $\mathrm{CO}_{2}$ & 1 & 38.151 & 38.151 & 256.225 & $<0.001 *$ \\
\hline & Ulva & 1 & 0.843 & 0.843 & 5.664 & $0.023^{*}$ \\
\hline & $\mathrm{CO}_{2} \times$ Ulva & 1 & 0.813 & 0.813 & 5.46 & $0.026^{*}$ \\
\hline & Residual & 32 & 4.765 & 0.149 & & \\
\hline & Total & 35 & 44.571 & 1.273 & & \\
\hline \multirow{5}{*}{$\begin{array}{c}\text { Crassostrea } \\
\text { virginica } \\
\text { (large) }\end{array}$} & $\mathrm{CO}_{2}$ & 1 & 7.276 & 7.276 & 475.686 & $<0.001 *$ \\
\hline & Ulva & 1 & 0.139 & 0.139 & 9.094 & $0.005^{*}$ \\
\hline & $\mathrm{CO}_{2} \times U l v a$ & 1 & 0.115 & 0.115 & 7.531 & $0.009^{*}$ \\
\hline & Residual & 38 & 0.581 & 0.0153 & & \\
\hline & Total & 41 & 8.218 & 0.2 & & \\
\hline \multirow{5}{*}{$\begin{array}{l}\text { Argopecten } \\
\text { irradians } \\
\text { (small) }\end{array}$} & $\mathrm{CO}_{2}$ & 1 & 15.648 & 15.648 & 400.024 & $<0.001^{*}$ \\
\hline & Ulva & 1 & 0.328 & 0.328 & 8.377 & $0.007 *$ \\
\hline & $\mathrm{CO}_{2} \times U l v a$ & 1 & 0.143 & 0.143 & 3.652 & $0.066^{*}$ \\
\hline & Residual & 28 & 1.095 & 0.0391 & & \\
\hline & Total & 31 & 17.214 & 0.555 & & \\
\hline \multirow{5}{*}{$\begin{array}{l}\text { Argopecten } \\
\text { irradians } \\
\quad \text { (large) }\end{array}$} & $\mathrm{CO}_{2}$ & 1 & 25.536 & 25.536 & 3709.725 & $<0.001 *$ \\
\hline & Ulva & 1 & 0.152 & 0.152 & 22.138 & $<0.001 *$ \\
\hline & $\mathrm{CO}_{2} \times U l v a$ & 1 & 0.0893 & 0.0893 & 12.974 & $<0.001 *$ \\
\hline & Residual & 48 & 0.33 & 0.00688 & & \\
\hline & Total & 51 & 26.108 & 0.512 & & \\
\hline \multirow{5}{*}{$\begin{array}{l}\text { Mytilus } \\
\text { edulis }\end{array}$} & $\mathrm{CO}_{2}$ & 1 & 16.436 & 16.436 & 159.827 & $<0.001 *$ \\
\hline & Ulva & 1 & 0.488 & 0.488 & 4.743 & $0.037 *$ \\
\hline & $\mathrm{CO}_{2} \times U l v a$ & 1 & 0.282 & 0.282 & 2.738 & 0.108 \\
\hline & Residual & 32 & 3.291 & 0.103 & & \\
\hline & Total & 35 & 21.189 & 0.605 & & \\
\hline
\end{tabular}


Table S4. Two-way analyses of variance for shell length-based growth for smaller and larger cohorts of hard clams (Mercenaria mercenaria), Eastern oysters (Crassostrea virginica), bay scallops (Argopecten irradians), and blue mussels (Mytilus edulis). Asterisks next to p-values represent significant results.

\begin{tabular}{|c|c|c|c|c|c|c|}
\hline $\begin{array}{c}\text { Species (size } \\
\text { class) }\end{array}$ & $\begin{array}{c}\text { Source of } \\
\text { variation }\end{array}$ & DF & SS & MS & $\mathrm{F}$ & $\mathrm{P}$ \\
\hline \multirow{5}{*}{$\begin{array}{l}\text { Mercenaria } \\
\text { mercenaria } \\
\quad \text { (small) }\end{array}$} & $\mathrm{CO}_{2}$ & 1 & 0.00293 & 0.00293 & 82.534 & $<0.001 *$ \\
\hline & Ulva & 1 & 0.000358 & 0.000358 & 10.107 & $0.009 *$ \\
\hline & $\mathrm{CO}_{2} \times U l v a$ & 1 & $6.44 \mathrm{E}-05$ & $6.44 \mathrm{E}-05$ & 1.815 & 0.205 \\
\hline & Residual & 11 & 0.00039 & $3.55 \mathrm{E}-05$ & & \\
\hline & Total & 14 & 0.00354 & 0.000253 & & \\
\hline \multirow{5}{*}{$\begin{array}{l}\text { Mercenaria } \\
\text { mercenaria } \\
\quad(\text { large })\end{array}$} & $\mathrm{CO}_{2}$ & 1 & 0.00171 & 0.00171 & 26.255 & $<0.001 *$ \\
\hline & Ulva & 1 & 0.000668 & 0.000668 & 10.278 & $0.008 *$ \\
\hline & $\mathrm{CO}_{2} \times$ Ulva & 1 & 0.000153 & 0.000153 & 2.348 & 0.154 \\
\hline & Residual & 11 & 0.000715 & 0.000065 & & \\
\hline & Total & 14 & 0.003 & 0.000214 & & \\
\hline \multirow{5}{*}{$\begin{array}{l}\text { Crassostrea } \\
\text { virginica } \\
\text { (small) }\end{array}$} & $\mathrm{CO}_{2}$ & 1 & 0.00395 & 0.00395 & 10.536 & $0.009 *$ \\
\hline & Ulva & 1 & 0.0022 & 0.0022 & 5.879 & $0.036^{*}$ \\
\hline & $\mathrm{CO}_{2} \times U l v a$ & 1 & 0.000368 & 0.000368 & 0.983 & 0.345 \\
\hline & Residual & 10 & 0.00375 & 0.000375 & & \\
\hline & Total & 13 & 0.01 & 0.000769 & & \\
\hline \multirow{5}{*}{$\begin{array}{l}\text { Crassostrea } \\
\text { virginica } \\
\text { (large) }\end{array}$} & $\mathrm{CO}_{2}$ & 1 & 0.0327 & 0.0327 & 34.363 & $<0.001 *$ \\
\hline & Ulva & 1 & 0.0111 & 0.0111 & 11.647 & $0.011^{*}$ \\
\hline & $\mathrm{CO}_{2} \times U l v a$ & 1 & 0.00204 & 0.00204 & 2.148 & 0.186 \\
\hline & Residual & 7 & 0.00666 & 0.000951 & & \\
\hline & Total & 10 & 0.0523 & 0.00523 & & \\
\hline \multirow{5}{*}{$\begin{array}{l}\text { Argopecten } \\
\text { irradians } \\
\text { (small) }\end{array}$} & $\mathrm{CO}_{2}$ & 1 & 0.00242 & 0.00242 & 31.308 & $<0.001 *$ \\
\hline & Ulva & 1 & 0.000917 & 0.000917 & 11.841 & $0.005^{*}$ \\
\hline & $\mathrm{CO}_{2} \times U l v a$ & 1 & 0.000303 & 0.000303 & 3.91 & 0.071 \\
\hline & Residual & 12 & 0.000929 & $7.74 \mathrm{E}-05$ & & \\
\hline & Total & 15 & 0.00457 & 0.000305 & & \\
\hline \multirow{5}{*}{$\begin{array}{c}\text { Argopecten } \\
\text { irradians } \\
\text { (large) }\end{array}$} & $\mathrm{CO}_{2}$ & 1 & 0.0035 & 0.0035 & 37.967 & $<0.001 *$ \\
\hline & Ulva & 1 & 0.001 & 0.001 & 10.852 & $0.013^{*}$ \\
\hline & $\mathrm{CO}_{2} \times U l v a$ & 1 & 0.000236 & 0.000236 & 2.561 & 0.154 \\
\hline & Residual & 7 & 0.000645 & $9.22 \mathrm{E}-05$ & & \\
\hline & Total & 10 & 0.00579 & 0.000579 & & \\
\hline \multirow{5}{*}{$\begin{array}{l}\text { Mytilus } \\
\text { edulis }\end{array}$} & $\mathrm{CO}_{2}$ & 1 & 0.000439 & 0.000439 & 2.93 & 0.118 \\
\hline & Ulva & 1 & 0.000786 & 0.000786 & 5.246 & $0.045^{*}$ \\
\hline & $\mathrm{CO}_{2} \times$ Ulva & 1 & $2.95 \mathrm{E}-05$ & $2.95 \mathrm{E}-05$ & 0.196 & 0.667 \\
\hline & Residual & 10 & 0.0015 & 0.00015 & & \\
\hline & Total & 13 & 0.0028 & 0.000215 & & \\
\hline
\end{tabular}


Table S5. Two-way analyses of variance for shell weight-based growth for smaller and larger cohorts of hard clams (Mercenaria mercenaria), Eastern oysters (Crassostrea virginica), bay scallops (Argopecten irradians), and blue mussels (Mytilus edulis). Asterisks next to p-values represent significant results.

\begin{tabular}{|c|c|c|c|c|c|c|}
\hline $\begin{array}{c}\text { Species (size } \\
\text { class) }\end{array}$ & $\begin{array}{l}\text { Source of } \\
\text { Variation }\end{array}$ & DF & SS & MS & $\mathrm{F}$ & $P$ \\
\hline \multirow{5}{*}{$\begin{array}{l}\text { Mercenaria } \\
\text { mercenaria } \\
\quad \text { (small) }\end{array}$} & $\mathrm{CO}_{2}$ & 1 & 0.0135 & 0.0135 & 21.016 & $<0.001 *$ \\
\hline & Ulva & 1 & 0.00405 & 0.00405 & 6.318 & $0.027 *$ \\
\hline & $\mathrm{CO}_{2} \times$ Ulva & 1 & 0.000164 & 0.000164 & 0.255 & 0.623 \\
\hline & Residual & 12 & 0.00769 & 0.000641 & & \\
\hline & Total & 15 & 0.0254 & 0.00169 & & \\
\hline \multirow{5}{*}{$\begin{array}{l}\text { Mercenaria } \\
\text { mercenaria } \\
\quad(\text { large })\end{array}$} & $\mathrm{CO}_{2}$ & 1 & 0.219 & 0.219 & 16.719 & $0.003^{*}$ \\
\hline & Ulva & 1 & 0.256 & 0.256 & 19.553 & $0.002 *$ \\
\hline & $\mathrm{CO}_{2} \times$ Ulva & 1 & 0.0489 & 0.0489 & 3.731 & 0.085 \\
\hline & Residual & 9 & 0.118 & 0.0131 & & \\
\hline & Total & 12 & 0.612 & 0.051 & & \\
\hline \multirow{5}{*}{$\begin{array}{l}\text { Crassostrea } \\
\text { virginica } \\
\text { (small) }\end{array}$} & $\mathrm{CO}_{2}$ & 1 & 0.0341 & 0.0341 & 8.801 & $0.013 *$ \\
\hline & Ulva & 1 & 0.000106 & 0.000106 & 0.0274 & 0.871 \\
\hline & $\mathrm{CO}_{2} \times$ Ulva & 1 & 0.000373 & 0.000373 & 0.0961 & 0.762 \\
\hline & Residual & 11 & 0.0427 & 0.00388 & & \\
\hline & Total & 14 & 0.0779 & 0.00557 & & \\
\hline \multirow{5}{*}{$\begin{array}{c}\text { Crassostrea } \\
\text { virginica } \\
\text { (large) }\end{array}$} & $\mathrm{CO}_{2}$ & 1 & 18.779 & 18.779 & 0.907 & 0.373 \\
\hline & Ulva & 1 & 33.088 & 33.088 & 1.598 & 0.247 \\
\hline & $\mathrm{CO}_{2} \times U l v a$ & 1 & 21.713 & 21.713 & 1.049 & 0.340 \\
\hline & Residual & 7 & 144.951 & 20.707 & & \\
\hline & Total & 10 & 223.95 & 22.395 & & \\
\hline \multirow{5}{*}{$\begin{array}{l}\text { Argopecten } \\
\text { irradians } \\
\text { (small) }\end{array}$} & $\mathrm{CO}_{2}$ & 1 & 0.119 & 0.119 & 9.587 & $0.009 *$ \\
\hline & Ulva & 1 & 0.00428 & 0.00428 & 0.344 & 0.569 \\
\hline & $\mathrm{CO}_{2} \times$ Ulva & 1 & 0.00553 & 0.00553 & 0.444 & 0.518 \\
\hline & Residual & 12 & 0.149 & 0.0124 & & \\
\hline & Total & 15 & 0.278 & 0.0186 & & \\
\hline \multirow{5}{*}{$\begin{array}{l}\text { Argopecten } \\
\text { irradians } \\
\quad \text { (large) }\end{array}$} & $\mathrm{CO}_{2}$ & 1 & 1.172 & 1.172 & 0.305 & 0.600 \\
\hline & Ulva & 1 & 3.929 & 3.929 & 1.024 & 0.351 \\
\hline & $\mathrm{CO}_{2} \times U l v a$ & 1 & 0.00124 & 0.00124 & 0.000323 & 0.986 \\
\hline & Residual & 6 & 23.015 & 3.836 & & \\
\hline & Total & 9 & 29.223 & 3.247 & & \\
\hline \multirow{5}{*}{$\begin{array}{l}\text { Mytilus } \\
\text { edulis }\end{array}$} & $\mathrm{CO}_{2}$ & 1 & 0.0113 & 0.0113 & 2.153 & 0.168 \\
\hline & Ulva & 1 & 0.0282 & 0.0282 & 5.392 & $0.039 *$ \\
\hline & $\mathrm{CO}_{2} \times$ Ulva & 1 & 0.00109 & 0.00109 & 0.209 & 0.656 \\
\hline & Residual & 12 & 0.0628 & 0.00523 & & \\
\hline & Total & 15 & 0.103 & 0.00689 & & \\
\hline
\end{tabular}


Table S6. Two-way analyses of variance for tissue weight-based growth for smaller and larger cohorts of hard clams (Mercenaria mercenaria), Eastern oysters (Crassostrea virginica), bay scallops (Argopecten irradians), and blue mussels (Mytilus edulis). Asterisks next to p-values represent significant results.

\begin{tabular}{|c|c|c|c|c|c|c|}
\hline $\begin{array}{c}\text { Species (size } \\
\text { class) }\end{array}$ & $\begin{array}{l}\text { Source of } \\
\text { Variation }\end{array}$ & DF & SS & MS & $\mathrm{F}$ & $\mathrm{P}$ \\
\hline \multirow{5}{*}{$\begin{array}{l}\text { Mercenaria } \\
\text { mercenaria } \\
\quad \text { (small) }\end{array}$} & $\mathrm{CO}_{2}$ & 1 & 0.000121 & 0.000121 & 8.339 & $0.018^{*}$ \\
\hline & Ulva & 1 & 0.000146 & 0.000146 & 10.006 & $0.011^{*}$ \\
\hline & $\mathrm{CO}_{2} \times U l v a$ & 1 & $2.66 \mathrm{E}-07$ & $2.66 \mathrm{E}-07$ & 0.0183 & 0.895 \\
\hline & Residual & 9 & 0.000131 & $1.46 \mathrm{E}-05$ & & \\
\hline & Total & 12 & 0.000382 & $3.18 \mathrm{E}-05$ & & \\
\hline \multirow{5}{*}{$\begin{array}{l}\text { Mercenaria } \\
\text { mercenaria } \\
\quad(\text { large })\end{array}$} & $\mathrm{CO}_{2}$ & 1 & 0.000996 & 0.000996 & 23.216 & $<0.001 *$ \\
\hline & Ulva & 1 & 0.000588 & 0.000588 & 13.703 & $0.005^{*}$ \\
\hline & $\mathrm{CO}_{2} \times U l v a$ & 1 & $1.19 \mathrm{E}-05$ & $1.19 \mathrm{E}-05$ & 0.276 & 0.612 \\
\hline & Residual & 9 & 0.000386 & 4.29E-05 & & \\
\hline & Total & 12 & 0.00189 & 0.000157 & & \\
\hline \multirow{5}{*}{$\begin{array}{c}\text { Crassostrea } \\
\text { virginica } \\
\text { (small) }\end{array}$} & $\mathrm{CO}_{2}$ & 1 & 0.00177 & 0.00177 & 11.614 & $0.007 *$ \\
\hline & Ulva & 1 & 0.000183 & 0.000183 & 1.201 & 0.299 \\
\hline & $\mathrm{CO}_{2} \times U l v a$ & 1 & 8.87E-07 & 8.87E-07 & 0.00584 & 0.941 \\
\hline & Residual & 10 & 0.00152 & 0.000152 & & \\
\hline & Total & 13 & 0.00334 & 0.000257 & & \\
\hline \multirow{5}{*}{$\begin{array}{l}\text { Crassostrea } \\
\text { virginica } \\
\text { (large) }\end{array}$} & $\mathrm{CO}_{2}$ & 1 & 3.141 & 3.141 & 1.034 & 0.356 \\
\hline & Ulva & 1 & 0.549 & 0.549 & 0.181 & 0.688 \\
\hline & $\mathrm{CO}_{2} \times$ Ulva & 1 & 26.628 & 26.628 & 8.768 & $0.031 *$ \\
\hline & Residual & 5 & 15.185 & 3.037 & & \\
\hline & Total & 8 & 43.69 & 5.461 & & \\
\hline \multirow{5}{*}{$\begin{array}{c}\text { Argopecten } \\
\text { irradians } \\
\text { (small) }\end{array}$} & $\mathrm{CO}_{2}$ & 1 & 0.00322 & 0.00322 & 8.107 & $0.016^{*}$ \\
\hline & Ulva & 1 & 0.000807 & 0.000807 & 2.031 & 0.182 \\
\hline & $\mathrm{CO}_{2} \times U l v a$ & 1 & $3.30 \mathrm{E}-06$ & $3.30 \mathrm{E}-06$ & 0.00831 & 0.929 \\
\hline & Residual & 11 & 0.00437 & 0.000398 & & \\
\hline & Total & 14 & 0.00868 & 0.00062 & & \\
\hline \multirow{5}{*}{$\begin{array}{l}\text { Argopecten } \\
\text { irradians } \\
\quad(\text { large })\end{array}$} & $\mathrm{CO}_{2}$ & 1 & 0.517 & 0.517 & 10.081 & 0.034 \\
\hline & Ulva & 1 & 0.796 & 0.796 & 15.505 & $0.017 *$ \\
\hline & $\mathrm{CO}_{2} \times U l v a$ & 1 & 0.0685 & 0.0685 & 1.334 & 0.312 \\
\hline & Residual & 4 & 0.205 & 0.0513 & & \\
\hline & Total & 7 & 1.587 & 0.227 & & \\
\hline \multirow{5}{*}{$\begin{array}{l}\text { Mytilus } \\
\text { edulis }\end{array}$} & $\mathrm{CO}_{2}$ & 1 & 0.000337 & 0.000337 & 2.136 & 0.172 \\
\hline & Ulva & 1 & 0.000794 & 0.000794 & 5.026 & $0.047 *$ \\
\hline & $\mathrm{CO}_{2} \times U l v a$ & 1 & $5.96 \mathrm{E}-05$ & $5.96 \mathrm{E}-05$ & 0.377 & 0.552 \\
\hline & Residual & 11 & 0.00174 & 0.000158 & & \\
\hline & Total & 14 & 0.00303 & 0.000216 & & \\
\hline
\end{tabular}


Table S7. Tukey Honest Significant Difference tests for shell length-based growth for tissue weightbased growth for smaller and larger cohorts of hard clams (Mercenaria mercenaria), Eastern oysters (Crassostrea virginica), bay scallops (Argopecten irradians), and blue mussels (Mytilus edulis). Asterisks next to p-values represent significant results.

\begin{tabular}{|c|c|c|c|c|c|}
\hline Species (cohort) & Treatment & Difference & Lower & Upper & P-value \\
\hline \multirow{6}{*}{$\begin{array}{c}\text { Mercenaria } \\
\text { mercenaria (small) }\end{array}$} & $\mathrm{CO}_{2}$ vs. Control & -0.032 & -0.046 & -0.019 & $<0.001^{*}$ \\
\hline & Ulva vs. Control & 0.006 & -0.007 & 0.018 & 0.554 \\
\hline & $\mathrm{CO}_{2} /$ Ulva vs. Control & -0.018 & -0.031 & -0.006 & $0.005^{*}$ \\
\hline & Ulva vs. $\mathrm{CO}_{2}$ & 0.038 & 0.024 & 0.052 & $<0.001 *$ \\
\hline & $\mathrm{CO}_{2} /$ Ulva vs. $\mathrm{CO}_{2}$ & 0.014 & 0.000 & 0.028 & $0.044 *$ \\
\hline & $\mathrm{CO}_{2} /$ Ulva vs. Ulva & -0.024 & -0.037 & -0.011 & $0.001 *$ \\
\hline \multirow{6}{*}{$\begin{array}{c}\text { Mercenaria } \\
\text { mercenaria (large) }\end{array}$} & $\mathrm{CO}_{2}$ vs. Control & -0.005 & -0.022 & 0.012 & 0.796 \\
\hline & Ulva vs. Control & 0.030 & 0.012 & 0.048 & $0.002 *$ \\
\hline & $\mathrm{CO}_{2} /$ Ulva vs. Control & 0.002 & -0.015 & 0.019 & 0.984 \\
\hline & Ulva vs. $\mathrm{CO}_{2}$ & 0.035 & 0.017 & 0.053 & $0.001 *$ \\
\hline & $\mathrm{CO}_{2} /$ Ulva vs. $\mathrm{CO}_{2}$ & 0.007 & -0.010 & 0.024 & 0.598 \\
\hline & $\mathrm{CO}_{2} /$ Ulva vs. Ulva & -0.028 & -0.046 & -0.010 & $0.003 *$ \\
\hline \multirow{6}{*}{$\begin{array}{c}\text { Crassostrea } \\
\text { virginica (small) }\end{array}$} & $\mathrm{CO}_{2}$ vs. Control & -0.027 & -0.071 & 0.017 & 0.289 \\
\hline & Ulva vs. Control & 0.049 & 0.002 & 0.096 & $0.040 *$ \\
\hline & $\mathrm{CO}_{2} /$ Ulva vs. Control & -0.012 & -0.059 & 0.035 & 0.861 \\
\hline & Ulva vs. $\mathrm{CO}_{2}$ & 0.076 & 0.029 & 0.123 & $0.003 *$ \\
\hline & $\mathrm{CO}_{2} /$ Ulva vs. $\mathrm{CO}_{2}$ & 0.015 & -0.032 & 0.062 & 0.767 \\
\hline & $\mathrm{CO}_{2} /$ Ulva vs. Ulva & -0.061 & -0.111 & -0.011 & $0.018 *$ \\
\hline \multirow{6}{*}{$\begin{array}{c}\text { Crassostrea } \\
\text { virginica (large) }\end{array}$} & $\mathrm{CO}_{2}$ vs. Control & -0.138 & -0.222 & -0.055 & $0.004 *$ \\
\hline & Ulva vs. Control & 0.037 & -0.056 & 0.130 & 0.587 \\
\hline & $\mathrm{CO}_{2} /$ Ulva vs. Control & -0.046 & -0.130 & 0.037 & 0.333 \\
\hline & Ulva vs. $\mathrm{CO}_{2}$ & 0.175 & 0.082 & 0.268 & $0.002 *$ \\
\hline & $\mathrm{CO}_{2} /$ Ulva vs. $\mathrm{CO}_{2}$ & 0.092 & 0.009 & 0.176 & $0.032 *$ \\
\hline & $\mathrm{CO}_{2}$ /Ulva vs. Ulva & -0.083 & -0.176 & 0.010 & 0.080 \\
\hline \multirow{6}{*}{$\begin{array}{c}\text { Argopecten } \\
\text { irradians (small) }\end{array}$} & $\mathrm{CO}_{2}$ vs. Control & -0.033 & -0.052 & -0.015 & $0.001 *$ \\
\hline & Ulva vs. Control & 0.006 & -0.012 & 0.025 & 0.733 \\
\hline & $\mathrm{CO}_{2} /$ Ulva vs. Control & -0.009 & -0.028 & 0.009 & 0.455 \\
\hline & Ulva vs. $\mathrm{CO}_{2}$ & 0.040 & 0.021 & 0.058 & $<0.001 *$ \\
\hline & $\mathrm{CO}_{2} /$ Ulva vs. $\mathrm{CO}_{2}$ & 0.024 & 0.005 & 0.042 & $0.011 *$ \\
\hline & $\mathrm{CO}_{2} /$ Ulva vs. Ulva & -0.016 & -0.034 & 0.003 & 0.100 \\
\hline \multirow{6}{*}{$\begin{array}{c}\text { Argopecten } \\
\text { irradians (large) }\end{array}$} & $\mathrm{CO}_{2}$ vs. Control & -0.027 & -0.053 & -0.001 & $0.043^{*}$ \\
\hline & Ulva vs. Control & 0.029 & 0.003 & 0.055 & $0.032 *$ \\
\hline & $\mathrm{CO}_{2} /$ Ulva vs. Control & -0.017 & -0.046 & 0.012 & 0.300 \\
\hline & Ulva vs. $\mathrm{CO}_{2}$ & 0.056 & 0.030 & 0.082 & $0.001 *$ \\
\hline & $\mathrm{CO}_{2} /$ Ulva vs. $\mathrm{CO}_{2}$ & 0.010 & -0.019 & 0.039 & 0.681 \\
\hline & $\mathrm{CO}_{2} /$ Ulva vs. Ulva & -0.046 & -0.075 & -0.017 & $0.005^{*}$ \\
\hline \multirow{6}{*}{ Mytilus edulis } & $\mathrm{CO}_{2}$ vs. Control & 0.014 & -0.012 & 0.041 & 0.398 \\
\hline & Ulva vs. Control & 0.018 & -0.011 & 0.047 & 0.275 \\
\hline & $\mathrm{CO}_{2} /$ Ulva vs. Control & 0.026 & -0.002 & 0.055 & 0.072 \\
\hline & Ulva vs. $\mathrm{CO}_{2}$ & 0.004 & -0.025 & 0.032 & 0.976 \\
\hline & $\mathrm{CO}_{2} /$ Ulva vs. $\mathrm{CO}_{2}$ & 0.012 & -0.016 & 0.041 & 0.580 \\
\hline & $\mathrm{CO}_{2} /$ Ulva vs. Ulva & 0.008 & -0.022 & 0.039 & 0.835 \\
\hline
\end{tabular}


Table S8. Tukey Honest Significant Difference tests for shell weight-based growth for tissue weightbased growth for smaller and larger cohorts of hard clams (Mercenaria mercenaria), Eastern oysters (Crassostrea virginica), bay scallops (Argopecten irradians), and blue mussels (Mytilus edulis). Asterisks next to p-values represent significant results.

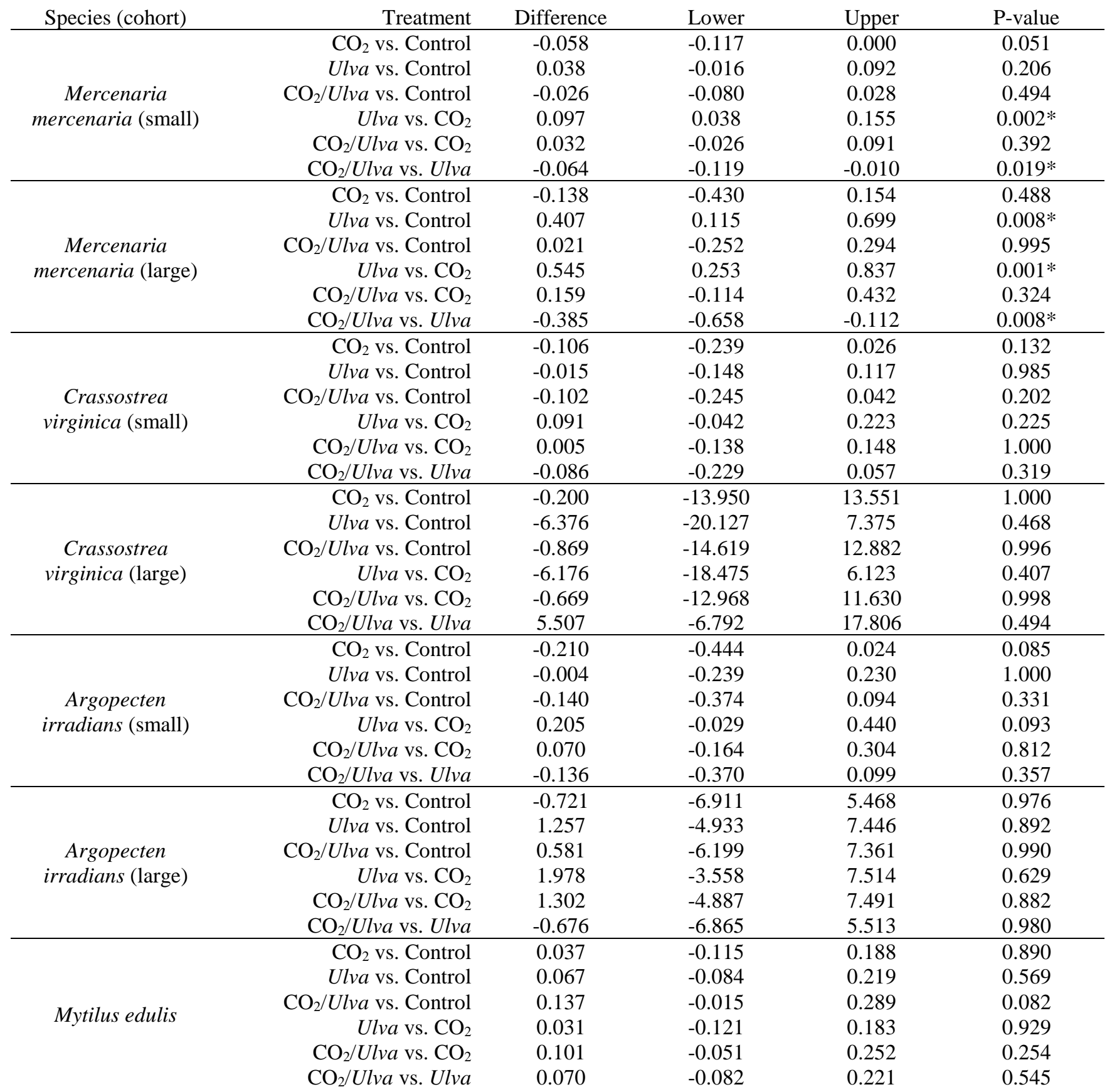


Table S9. Tukey Honest Significant Difference tests for tissue weight-based growth for tissue weightbased growth for smaller and larger cohorts of hard clams (Mercenaria mercenaria), Eastern oysters (Crassostrea virginica), bay scallops (Argopecten irradians), and blue mussels (Mytilus edulis). Asterisks next to p-values represent significant results.

\begin{tabular}{|c|c|c|c|c|c|}
\hline Species (cohort) & Treatment & Difference & Lower & Upper & $\mathrm{P}$-value \\
\hline \multirow{6}{*}{$\begin{array}{c}\text { Mercenaria } \\
\text { mercenaria (small) }\end{array}$} & $\mathrm{CO}_{2}$ vs. Control & -0.006 & -0.016 & 0.003 & 0.233 \\
\hline & Ulva vs. Control & 0.006 & -0.003 & 0.016 & 0.232 \\
\hline & $\mathrm{CO}_{2} /$ Ulva vs. Control & 0.001 & -0.009 & 0.010 & 0.997 \\
\hline & Ulva vs. $\mathrm{CO}_{2}$ & 0.013 & 0.003 & 0.023 & $0.011 *$ \\
\hline & $\mathrm{CO}_{2} /$ Ulva vs. $\mathrm{CO}_{2}$ & 0.007 & -0.002 & 0.016 & 0.143 \\
\hline & $\mathrm{CO}_{2} /$ Ulva vs. Ulva & -0.006 & -0.015 & 0.003 & 0.252 \\
\hline \multirow{6}{*}{$\begin{array}{c}\text { Mercenaria } \\
\text { mercenaria (large) }\end{array}$} & $\mathrm{CO}_{2}$ vs. Control & -0.016 & -0.033 & 0.001 & 0.070 \\
\hline & Ulva vs. Control & 0.015 & -0.002 & 0.031 & 0.098 \\
\hline & $\mathrm{CO}_{2} /$ Ulva vs. Control & -0.004 & -0.020 & 0.012 & 0.851 \\
\hline & Ulva vs. $\mathrm{CO}_{2}$ & 0.030 & 0.013 & 0.047 & $0.002 *$ \\
\hline & $\mathrm{CO}_{2} /$ Ulva vs. $\mathrm{CO}_{2}$ & 0.012 & -0.004 & 0.027 & 0.172 \\
\hline & $\mathrm{CO}_{2} /$ Ulva vs. Ulva & -0.019 & -0.034 & -0.003 & $0.022 *$ \\
\hline \multirow{6}{*}{$\begin{array}{c}\text { Crassostrea } \\
\text { virginica (small) }\end{array}$} & $\mathrm{CO}_{2}$ vs. Control & -0.023 & -0.052 & 0.006 & 0.127 \\
\hline & Ulva vs. Control & 0.007 & -0.022 & 0.036 & 0.887 \\
\hline & $\mathrm{CO}_{2} /$ Ulva vs. Control & -0.015 & -0.042 & 0.011 & 0.343 \\
\hline & Ulva vs. $\mathrm{CO}_{2}$ & 0.030 & -0.001 & 0.061 & 0.057 \\
\hline & $\mathrm{CO}_{2} / U l v a$ vs. $\mathrm{CO}_{2}$ & 0.008 & -0.021 & 0.037 & 0.840 \\
\hline & $\mathrm{CO}_{2} /$ Ulva vs. Ulva & -0.022 & -0.051 & 0.007 & 0.150 \\
\hline \multirow{6}{*}{$\begin{array}{c}\text { Crassostrea } \\
\text { virginica (large) }\end{array}$} & $\mathrm{CO}_{2}$ vs. Control & -4.693 & -11.124 & 1.737 & 0.141 \\
\hline & Ulva vs. Control & -2.992 & -8.862 & 2.878 & 0.341 \\
\hline & $\mathrm{CO}_{2} /$ Ulva vs. Control & -0.698 & -7.129 & 5.732 & 0.976 \\
\hline & Ulva vs. $\mathrm{CO}_{2}$ & 1.701 & -4.169 & 7.572 & 0.721 \\
\hline & $\mathrm{CO}_{2} /$ Ulva vs. $\mathrm{CO}_{2}$ & 3.995 & -2.435 & 10.425 & 0.219 \\
\hline & $\mathrm{CO}_{2} /$ Ulva vs. Ulva & 2.294 & -3.577 & 8.164 & 0.529 \\
\hline \multirow{6}{*}{$\begin{array}{c}\text { Argopecten } \\
\text { irradians (small) }\end{array}$} & $\mathrm{CO}_{2}$ vs. Control & -0.030 & -0.076 & 0.015 & 0.245 \\
\hline & Ulva vs. Control & -0.016 & -0.058 & 0.027 & 0.688 \\
\hline & $\mathrm{CO}_{2} /$ Ulva vs. Control & -0.044 & -0.087 & -0.002 & $0.040^{*}$ \\
\hline & Ulva vs. $\mathrm{CO}_{2}$ & 0.015 & -0.031 & 0.061 & 0.769 \\
\hline & $\mathrm{CO}_{2} /$ Ulva vs. $\mathrm{CO}_{2}$ & -0.014 & -0.060 & 0.032 & 0.801 \\
\hline & $\mathrm{CO}_{2} /$ Ulva vs. Ulva & -0.029 & -0.071 & 0.014 & 0.236 \\
\hline \multirow{6}{*}{$\begin{array}{c}\text { Argopecten } \\
\text { irradians (large) }\end{array}$} & $\mathrm{CO}_{2}$ vs. Control & -0.324 & -1.246 & 0.599 & 0.546 \\
\hline & Ulva vs. Control & 0.816 & -0.106 & 1.738 & 0.073 \\
\hline & $\mathrm{CO}_{2} /$ Ulva vs. Control & 0.122 & -0.800 & 1.044 & 0.945 \\
\hline & Ulva vs. $\mathrm{CO}_{2}$ & 1.139 & 0.217 & 2.061 & $0.025^{*}$ \\
\hline & $\mathrm{CO}_{2} /$ Ulva vs. $\mathrm{CO}_{2}$ & 0.446 & -0.476 & 1.368 & 0.332 \\
\hline & $\mathrm{CO}_{2} /$ Ulva vs. Ulva & -0.694 & -1.616 & 0.229 & 0.118 \\
\hline \multirow{6}{*}{ Mytilus edulis } & $\mathrm{CO}_{2}$ vs. Control & 0.006 & -0.021 & 0.032 & 0.922 \\
\hline & Ulva vs. Control & 0.011 & -0.018 & 0.040 & 0.692 \\
\hline & $\mathrm{CO}_{2} /$ Ulva vs. Control & 0.024 & -0.003 & 0.051 & 0.080 \\
\hline & Ulva vs. $\mathrm{CO}_{2}$ & 0.005 & -0.024 & 0.034 & 0.949 \\
\hline & $\mathrm{CO}_{2} /$ Ulva vs. $\mathrm{CO}_{2}$ & 0.019 & -0.008 & 0.045 & 0.212 \\
\hline & $\mathrm{CO}_{2} /$ Ulva vs. Ulva & 0.014 & -0.015 & 0.042 & 0.517 \\
\hline
\end{tabular}


Table S10. Two-way analyses of variance for total alkalinity for smaller and larger cohorts of hard clams (Mercenaria mercenaria), Eastern oysters (Crassostrea virginica), bay scallops (Argopecten irradians), and blue mussels (Mytilus edulis). Asterisks next to p-values represent significant results.

\begin{tabular}{|c|c|c|c|c|c|c|}
\hline $\begin{array}{c}\text { Species (size } \\
\text { class) }\end{array}$ & $\begin{array}{l}\text { Source of } \\
\text { Variation }\end{array}$ & DF & SS & MS & F & $\mathrm{P}$ \\
\hline \multirow{5}{*}{$\begin{array}{l}\text { Mercenaria } \\
\text { mercenaria } \\
\quad \text { (small) }\end{array}$} & $\mathrm{CO}_{2}$ & 1 & 21445.146 & 21445.146 & 80.677 & $<0.001 *$ \\
\hline & Ulva & 1 & 77.383 & 77.383 & 0.291 & 0.593 \\
\hline & $\mathrm{CO}_{2} \times$ Ulva & 1 & 1.263 & 1.263 & 0.00475 & 0.945 \\
\hline & Residual & 37 & 9835.142 & 265.815 & & \\
\hline & Total & 40 & 31702.957 & 792.574 & & \\
\hline \multirow{5}{*}{$\begin{array}{l}\text { Mercenaria } \\
\text { mercenaria } \\
\quad \text { (large) }\end{array}$} & $\mathrm{CO}_{2}$ & 1 & 12167.668 & 12167.668 & 78.766 & $<0.001 *$ \\
\hline & Ulva & 1 & 4868.925 & 4868.925 & 31.519 & $<0.001 *$ \\
\hline & $\mathrm{CO}_{2} \times U l v a$ & 1 & 298502.623 & 298502.623 & 1932.331 & $<0.001 *$ \\
\hline & Residual & 30 & 4634.341 & 154.478 & & \\
\hline & Total & 33 & 317210.57 & 9612.442 & & \\
\hline \multirow{5}{*}{$\begin{array}{c}\text { Crassostrea } \\
\text { virginica } \\
\text { (small) }\end{array}$} & $\mathrm{CO}_{2}$ & 1 & 94349.785 & 94349.785 & 70.625 & $<0.001 *$ \\
\hline & Ulva & 1 & 12338.03 & 12338.03 & 9.236 & $0.005^{*}$ \\
\hline & $\mathrm{CO}_{2} \times$ Ulva & 1 & 13725.68 & 13725.68 & 10.274 & $0.003 *$ \\
\hline & Residual & 32 & 42749.609 & 1335.925 & & \\
\hline & Total & 35 & 163163.104 & 4661.803 & & \\
\hline \multirow{5}{*}{$\begin{array}{c}\text { Crassostrea } \\
\text { virginica } \\
\text { (large) }\end{array}$} & $\mathrm{CO}_{2}$ & 1 & 365185.127 & 365185.127 & 1740.652 & $<0.001 *$ \\
\hline & Ulva & 1 & 450.572 & 450.572 & 2.148 & 0.151 \\
\hline & $\mathrm{CO}_{2} \times U l v a$ & 1 & 57698.523 & 57698.523 & 275.02 & $<0.001 *$ \\
\hline & Residual & 36 & 7552.724 & 209.798 & & \\
\hline & Total & 39 & 435434.308 & 11164.982 & & \\
\hline \multirow{5}{*}{$\begin{array}{l}\text { Argopecten } \\
\text { irradians } \\
\text { (small) }\end{array}$} & $\mathrm{CO}_{2}$ & 1 & 43126.683 & 43126.683 & 108.315 & $<0.001 *$ \\
\hline & Ulva & 1 & 73322.882 & 73322.882 & 184.155 & $<0.001 *$ \\
\hline & $\mathrm{CO}_{2} \times U l v a$ & 1 & 9647.501 & 9647.501 & 24.23 & $<0.001 *$ \\
\hline & Residual & 28 & 11148.464 & 398.159 & & \\
\hline & Total & 31 & 137245.529 & 4427.275 & & \\
\hline \multirow{5}{*}{$\begin{array}{l}\text { Argopecten } \\
\text { irradians } \\
\quad \text { (large) }\end{array}$} & $\mathrm{CO}_{2}$ & 1 & 52935.913 & 52935.913 & 521.079 & $<0.001 *$ \\
\hline & Ulva & 1 & 35280.605 & 35280.605 & 347.287 & $<0.001 *$ \\
\hline & $\mathrm{CO}_{2} \times U l v a$ & 1 & 14073.663 & 14073.663 & 138.535 & $<0.001 *$ \\
\hline & Residual & 48 & 4876.275 & 101.589 & & \\
\hline & Total & 51 & 107166.457 & 2101.303 & & \\
\hline \multirow{5}{*}{$\begin{array}{l}\text { Mytilus } \\
\text { edulis }\end{array}$} & $\mathrm{CO}_{2}$ & 1 & 39973.607 & 39973.607 & 37.22 & $<0.001 *$ \\
\hline & Ulva & 1 & 58814.526 & 58814.526 & 54.764 & $<0.001 *$ \\
\hline & $\mathrm{CO}_{2} \times$ Ulva & 1 & 26759.68 & 26759.68 & 24.917 & $<0.001 *$ \\
\hline & Residual & 32 & 34367.021 & 1073.969 & & \\
\hline & Total & 35 & 168107.844 & 4803.081 & & \\
\hline
\end{tabular}


Table S11. Two-way analyses of variance for $\mathrm{OH}^{-}$concentrations for smaller and larger cohorts of hard clams (Mercenaria mercenaria), eastern oysters (Crassostrea virginica), bay scallops (Argopecten irradians), and blue mussels (Mytilus edulis). Asterisks next to p-values represent significant results.

\begin{tabular}{|c|c|c|c|c|c|c|}
\hline $\begin{array}{c}\text { Species (size } \\
\text { class) }\end{array}$ & $\begin{array}{l}\text { Source of } \\
\text { Variation }\end{array}$ & DF & SS & MS & $\mathrm{F}$ & $\mathrm{P}$ \\
\hline \multirow{5}{*}{$\begin{array}{l}\text { Mercenaria } \\
\text { mercenaria } \\
\quad(\text { small })\end{array}$} & $\mathrm{CO}_{2}$ & 1 & 172.479 & 172.479 & 1029.599 & $<0.001 *$ \\
\hline & Ulva & 1 & 1.023 & 1.023 & 6.109 & $0.018^{*}$ \\
\hline & $\mathrm{CO}_{2} \times$ Ulva & 1 & 0.574 & 0.574 & 3.425 & 0.072 \\
\hline & Residual & 37 & 6.198 & 0.168 & & \\
\hline & Total & 40 & 179.932 & 4.498 & & \\
\hline \multirow{5}{*}{$\begin{array}{l}\text { Mercenaria } \\
\text { mercenaria } \\
\quad \text { (large) }\end{array}$} & $\mathrm{CO}_{2}$ & 1 & 56.09 & 56.09 & 911.005 & $<0.001 *$ \\
\hline & Ulva & 1 & 1.328 & 1.328 & 21.564 & $<0.001 *$ \\
\hline & $\mathrm{CO}_{2} \times U l v a$ & 1 & 1.118 & 1.118 & 18.159 & $<0.001 *$ \\
\hline & Residual & 30 & 1.847 & 0.0616 & & \\
\hline & Total & 33 & 59.195 & 1.794 & & \\
\hline \multirow{5}{*}{$\begin{array}{l}\text { Crassostrea } \\
\text { virginica } \\
\text { (small) }\end{array}$} & $\mathrm{CO}_{2}$ & 1 & 212.983 & 212.983 & 199.46 & $<0.001 *$ \\
\hline & Ulva & 1 & 7.62 & 7.62 & 7.136 & $0.012 *$ \\
\hline & $\mathrm{CO}_{2} \times$ Ulva & 1 & 6.629 & 6.629 & 6.208 & $0.018^{*}$ \\
\hline & Residual & 32 & 34.17 & 1.068 & & \\
\hline & Total & 35 & 261.401 & 7.469 & & \\
\hline \multirow{5}{*}{$\begin{array}{l}\text { Crassostrea } \\
\text { virginica } \\
\quad \text { (large) }\end{array}$} & $\mathrm{CO}_{2}$ & 1 & 49.25 & 49.25 & 464.178 & $<0.001 *$ \\
\hline & Ulva & 1 & 0.498 & 0.498 & 4.698 & $0.037^{*}$ \\
\hline & $\mathrm{CO}_{2} \times U l v a$ & 1 & 0.325 & 0.325 & 3.066 & 0.088 \\
\hline & Residual & 37 & 3.926 & 0.106 & & \\
\hline & Total & 40 & 54.324 & 1.358 & & \\
\hline \multirow{5}{*}{$\begin{array}{l}\text { Argopecten } \\
\text { irradians } \\
\text { (small) }\end{array}$} & $\mathrm{CO}_{2}$ & 1 & 67.22 & 67.22 & 264.944 & $<0.001 *$ \\
\hline & Ulva & 1 & 1.187 & 1.187 & 4.68 & $0.040^{*}$ \\
\hline & $\mathrm{CO}_{2} \times U l v a$ & 1 & 0.918 & 0.918 & 3.618 & 0.068 \\
\hline & Residual & 26 & 6.597 & 0.254 & & \\
\hline & Total & 29 & 77.278 & 2.665 & & \\
\hline \multirow{5}{*}{$\begin{array}{l}\text { Argopecten } \\
\text { irradians } \\
\text { (large) }\end{array}$} & $\mathrm{CO}_{2}$ & 1 & 103.532 & 103.532 & 2999.894 & $<0.001 *$ \\
\hline & Ulva & 1 & 0.181 & 0.181 & 5.255 & $0.026^{*}$ \\
\hline & $\mathrm{CO}_{2} \times$ Ulva & 1 & 0.0825 & 0.0825 & 2.39 & 0.129 \\
\hline & Residual & 48 & 1.657 & 0.0345 & & \\
\hline & Total & 51 & 105.452 & 2.068 & & \\
\hline \multirow{5}{*}{$\begin{array}{c}\text { Mytilus } \\
\text { edulis }\end{array}$} & $\mathrm{CO}_{2}$ & 1 & 72.466 & 72.466 & 111.682 & $<0.001 *$ \\
\hline & Ulva & 1 & 2.977 & 2.977 & 4.588 & $0.040^{*}$ \\
\hline & $\mathrm{CO}_{2} \times U l v a$ & 1 & 1.869 & 1.869 & 2.88 & 0.100 \\
\hline & Residual & 31 & 20.115 & 0.649 & & \\
\hline & Total & 34 & 103.361 & 3.04 & & \\
\hline
\end{tabular}


Table S12. One-way analysis of variance for growth rates of Ulva exposed to ambient and elevated $\mathrm{CO}_{2}$ conditions for June through November experiments.

\begin{tabular}{rccccc} 
Source of Variation & DF & SS & MS & F & P \\
\hline Between Groups & 1 & 0.0267 & 0.0267 & 4.264 & 0.041 \\
Residual & 111 & 0.695 & 0.00626 & & \\
Total & 112 & 0.722 & & &
\end{tabular}


Figure S1. Mean growth rates of Ulva exposed to ambient and elevated $\mathrm{CO}_{2}$ conditions for June through November experiments.

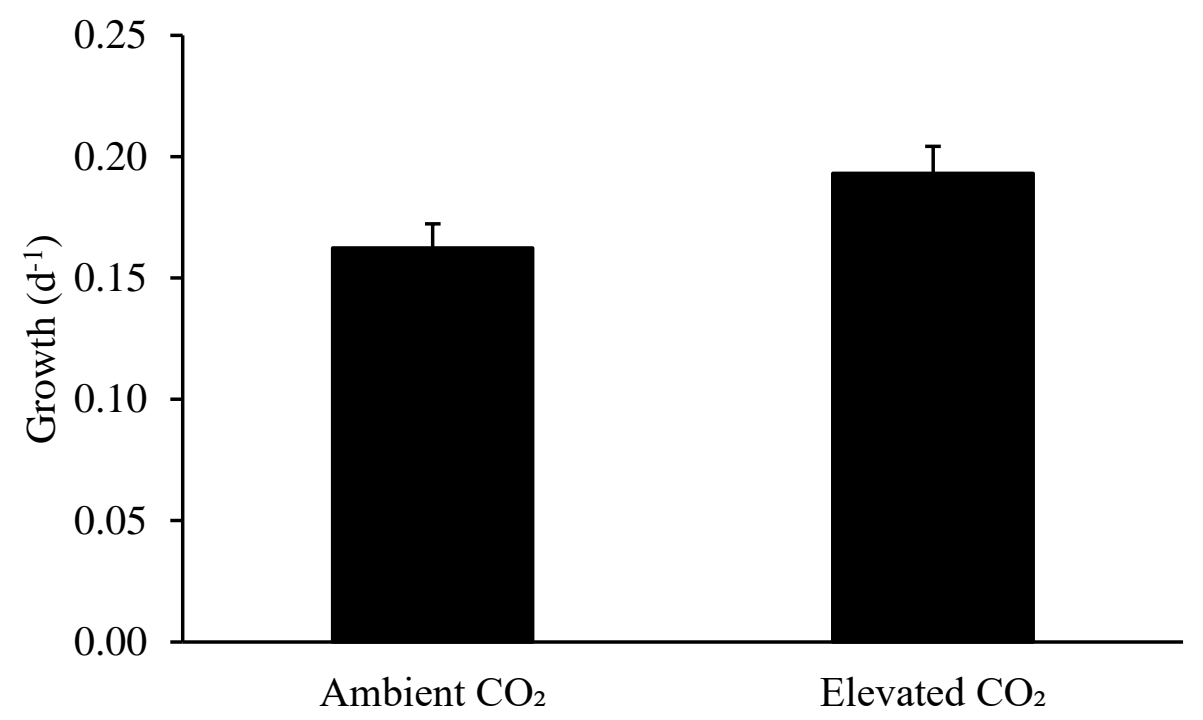

